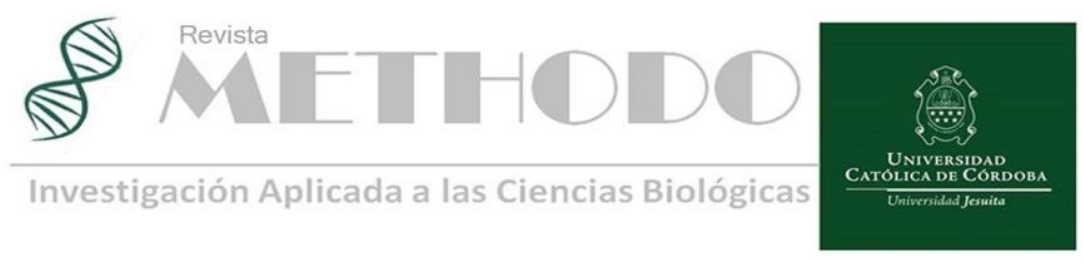

\title{
Genotoxicidad de la acrilamida y la glicidamida en Allium cepa
}

\section{Genotoxicity of acrylamide and glycidamide in Allium cepa}

\author{
Daniel Lerda ${ }^{1}$ \\ 1.Universidad Católica de Córdoba. Facultad de Ciencias de la Salud. Clínica Universitaria Reina Fabiola. Laboratorio de Genética Molecular. \\ Correspondencia: Daniel Enrique Lerda. Universidad Católica de Córdoba. Facultad de Ciencias de la Salud. Clínica Universitaria Reina Fabiola. Laboratorio de \\ Genética Molecular. E-mail: daniellerda@curf.ucc.edu.ar.
}

\section{Resumen}

INTRODUCCIÓN: la formación de acrilamida (AA) en alimentos que fueron sometidos a tratamiento térmico, fue un descubrimiento inesperado en abril del 2002 por la Universidad de Estocolmo y la Administración Nacional de Alimentos de Suecia (NFA).

Esta sustancia potencialmente tóxica y su metabolito glicidamida (GA) se forman en muchos alimentos cocidos a temperaturas elevadas.

OBJETIVO: en virtud de que no hay estudios de estas sustancias químicas, en células vegetales, el objetivo fue comprobar el efecto genotóxico de la AA y GA en células de Allium cepa.

MATERIALY MÉTODO: se determinó el efecto citotóxico a través del crecimiento de la raíz, actividad proliferativa y las aberraciones cromosómicas de la AA y GA en células meristemáticas de Allium cepa, expuestas a diferentes tiempos y concentraciones, evaluando el crecimiento de la raíz, la actividad proliferativa y las aberraciones cromosómicas.

RESULTADOS: GA causa una inhibición del crecimiento de la raíz cuya intensidad depende de la concentración en un medio dado y la AA no muestra diferencias estadísticamente significativas respecto al control. La GA bloquea el ciclo de división celular en una etapa previa a la mitosis y no ocurre lo mismo con la AA. En cuanto a los efectos clastogénicos la GA induce estos efectos en las raíces meristemáticas de Allium cepa, en cambio la AA no los produce.

CONCLUSIONES: en este estudio, aunque las concentraciones de AA y GA son mucho más altas que lo niveles de exposición alimentaria en humanos, la GA fue claramente genotóxica para células vegetales, a una determinada concentración, con una marcada citotoxicidad. Por lo tanto, nos permitió distinguir las diferencias entre AA y GA.

Palabras claves: acrilamida, glicidamida, Allium cepa, crecimiento radicular, actividad proliferativa, aberraciones cromosómicas.

\section{Abstract}

INTRODUCTION: the formation of acrylamide (AA) in foods that were sometimes heat treated was an unexpected discovery in April 2002, by Stockholm University and the Swedish National Food Administration (NFA). This potentially toxic substance and its metaboliteglycidamide (GA) form in many foods cooked at elevated temperatures.

OBJECTIVES: because there are no studies of these chemicals, in plant cells, the objective was to verify the genotoxic effect of AA and GA in Allium cepa cells. 
MATERIAL AND METHODS: to determine the cytotoxic effect through root growth, proliferative activity and chromosomal aberrations of acrylamide and glycidamide in Allium strain meristematic cells exposed to different times and antibodies, to evaluate root growth, proliferative activity and chromosomal aberrations.

RESULTS: GA causes an inhibition of root growth whose intensity depends on the concentration in a given medium and AA does not show statistically significant differences with respect to the control. GA blocks the cell division cycle at a stage prior to mitosis, and the same does not occur with AA. Regarding the clastogenic effects, GA induces these effects in the meristematic roots of Allium strain, whereas AA does not produce them. CONCLUSIONS: in this study, although AA and GA concentrations are much higher than levels of dietary exposure in humans, GA was clearly genotoxic to plant cells, at a certain concentration, with marked cytotoxicity. For the therefore, it allowed us to distinguish the differences between AA and GA.

Keywords: acrylamide, glycidamide, Allium cepa, root growth, proliferative activity, chromosomal aberrations.

\section{Introducción}

Son bien conocidas algunas sustancias químicas producto del proceso tecnológico de los alimentos, ellas son las aminas heterocíclicas, los hidrocarburos aromáticos policíclicos, las nitrosaminas y actualmente se incorporó la acrilamida (AA) ${ }^{1}$. Esta sustancia química es un conocido intermediario químico utilizado en la producción y síntesis de la poliacrilamida, que es utilizada como floculante para clarificar el agua en el tratamiento de las mismas para consumo humano, aguas de desecho como así también el tratamiento de aguas industriales de residuos sólidos antes del vertido o reutilización ${ }^{2}$. Otros usos importantes de la AA son la estabilización del suelo, la lechada para reparar alcantarillas y los geles de acrilamida en los laboratorios de biotecnología $^{3}$. Químicamente, la AA es un compuesto de bajo peso molecular soluble en agua (MW 79.01) formado por un doble enlace etílico reactivo unido a un grupo carboxiamida. Este tóxico, en exposición a altas concentraciones causa daño en el sistema nervioso central y ha sido clasificado por distintas agencias del mundo como carcinógeno o posiblemente carcinógeno: la Agencia Internacional para Investigación en Cáncer (IARC) "probable carcinógeno para humanos" (Clase 2A); Unión Europea, como carcinógeno, categoría 2 además, como mutágeno en la categoría 2 y tóxico para la reproducción en la categoría 3; Noruega como carcinógeno de alta potencia ${ }^{4-6}$.

La formación de AA en alimentos que fueron sometidos a un tratamiento térmico fue un descubrimiento inesperado en abril del 2002, por la Universidad de Estocolmo y la Administración Nacional de Alimentos de Suecia (NFA). Ellos determinaron que esta sustancia potencialmente tóxica se forma en muchos alimentos cocidos a temperaturas elevadas ${ }^{7,8}$.
La reacción de Maillard es la vía principal para la formación de AA: factores importantes son la presencia de sus precursores en materias primas (asparagina libre y azúcar reductora, glucosa y fructosa) y la magnitud de la carga de calor aplicado durante la producción de alimentos (combinación de tiempo y temperatura) ${ }^{9,10} \mathrm{Se}$ sabe que la selección de variedades y las condiciones ambientales modifican la concentración de precursores de la AA. Además, las condiciones de procesamiento y la actividad hídrica de los alimentos también pueden desempeñar un papel clave.

Se encuentra en alimentos como papas fritas o al horno, pan y productos de panadería como galletitas, cereales de desayuno, almendras tostadas y café ${ }^{11}$. En galletitas se han detectados niveles entre 12 y $1100 \mu \mathrm{g} / \mathrm{Kg}$ dependiendo, entre otros factores, del leudante químico utilizado, siendo mayor la generación de AA con bicarbonato de amonio ${ }^{12}$.

Por otra parte, la AA puede reaccionar con otros constituyentes de los alimentos; permanece sin cambios durante un año en cereales de desayuno, y casi sin cambios en el cacao, pero se pierde considerablemente durante el almacenamiento en el café soluble o tostado ${ }^{13}$. En los alimentos hervidos no se produce $\mathrm{AA}^{14}$. La temperatura y el tiempo de calentamiento influyen en la cantidad de AA presente ${ }^{15}$; a mayor temperatura y/o tiempo de calentamiento, mayor producción de AA, aunque en alimentos sometidos durante tiempos prolongados a altas temperaturas se observó una disminución debida a procesos de degradación o polimerización ${ }^{16-17}$.

La papa contiene pequeñas cantidades de azúcares reductores, que se incrementan si se expone la papa cruda a temperaturas menores a $10^{\circ} \mathrm{C}$. Por esta razón, no se debe guardar las papas a estas temperaturas. Las papas cortadas se pueden sumergir en agua fría o tibia unos $15 \mathrm{~min}$ para extraer los azúcares y la asparagina de la 
superficie sin eliminar el almidón ${ }^{18}$.Durante la fritura o el horneado de un alimento, la superficie se deshidrata alcanzando altas temperaturas, lo que favorece la formación de AA. Es decir que el contenido de AA es mayor en alimentos con mayor superficie expuesta ${ }^{16}$. Además, los fumadores de tabaco están muy expuestos a la AA. Bergmark ${ }^{19}$ determinó el nivel de aductos de AA en muestras de sangre de fumadores y no fumadores que trabajan con geles de poliacrilamida para la electroforesis. También Pere $^{20}$ midió niveles sorprendentemente altos de AA en el café. Estas primeras observaciones en el año 2000 fueron ampliamente ignoradas.

El metabolismo de AA en el organismo humano se produce mediante conjugación con glutatión reducido (GSH) que resulta en la excreción urinaria de un ácido mercaptúrico conjugado, o mediante epoxidación, presumiblemente mediado por citocromo P450 (CYP2E1), para producir el epóxido genotóxico glicidamida (GA). La GA puede metabolizarse mediante conjugación con GSH o someterse a hidrólisis del grupo epóxido por epóxido hidrolasa (EPHX) para formar gliceramida, que también se excreta en la orina ${ }^{21-24}$. Además, la actividad biológica de la AA también está mediada por su metabolito GA, que aparte de generar aductos de proteínas, tiene una alta afinidad con el ADN dando lugar a aductos de ADN. Por el contrario, la AA tiene una capacidad bastante débil para unirse al $\mathrm{ADN}^{25}$.

En estudios epidemiológicos de personas expuestas ocupacionalmente a la AA se observaron efectos neurotóxicos caracterizados por ataxia y debilidad de los músculos esqueléticos distales.

El manejo de riesgo de AA en productos alimenticios ha sido, desde su conocimiento, de carácter voluntario entre entidades gubernamentales, especialmente en Europa y Estados Unidos. Un ejemplo de ello es la creación de "Acrylamide Toolbox"26, sitio web en el que se puede encontrar información desde los contenidos en alimentos, hasta las estrategias de mitigación, con la finalidad de que el problema sea tratado de manera más relevante y acogido por los centros de investigación y desarrollo de la industria alimenticia ${ }^{27}$.Los estudios a largo plazo en modelos de roedores han demostrado que la AA es cancerígeno en diferentes órganos ${ }^{27}$. Sin embargo, no son consistentes. Se encontró evidencia de un mayor riesgo de cáncer entre los trabajadores expuesto a AA. Además, la asociación del mayor riesgo de cáncer humano por consumo dietético de AA sigue siendo una cuestión de discusión ${ }^{28}$. Si bien algunos estudios han encontrado asociaciones significativas entre la exposición oral a AA y el cáncer, otros no pudieron probar tal relación. Respecto a la genotoxicidad de la AA, esta ha sido ampliamente estudiada en distintos sistemas, pero no en células vegetales como en el test de Allium cepa. Entre los estudios se puede mencionar la inducción de mutación en bacterias, pero su metabolito, la GA, lo hace en ausencia de un sistema metabólico exógeno ${ }^{29}$. La AA induce mutaciones letales y somáticas recesivas ligadas al sexo en Drosophila. Induce mutaciones genéticas, aberraciones cromosómicas estructurales, intercambio de cromátidas hermanas y alteraciones mitóticas en células de mamíferos in vitro en presencia o ausencia de sistemas metabólicos exógenos. Se indujeron aberraciones cromosómicas y micronúcleos en la médula ósea de ratón y en células premeióticas y posmeióticas en una relación de dosis- respuesta lineal. La AA induce la síntesis de ADN no programada en espermatocitos de rata in vivo, pero aparentemente no en hepatocitos de rata. La GA indujo la síntesis de ADN no programada en hepatocitos de rata in vitro. Además, la AA induce la transformación en células de mamíferos cultivadas ${ }^{29-34}$. Por otra parte, ambos agentes reaccionan directamente con la hemoglobina in vivo, pero los aductos de ADN resultan solo de la formación de $\mathrm{GA}^{29-30}$.

El test de Allium cepa, por sus características, lo convierten en un excelente modelo genético para evaluar contaminantes ambientales y puede usarse para monitorear agentes tóxicos químicos y físicos. La aplicación de Allium cepa no solo se debe a la sensibilidad para detectar mutágenos, sino también a la posibilidad de evaluar varios puntos finales genéticos, desde la mutación puntual hasta las aberraciones cromosómicas (CA) en las células meristemáticas, así como la generación $\mathrm{F}^{35-37}$. La prueba de Allium cepa se considera una excelente presentación de la prueba biológica in vivo, donde las raíces crecen en contacto directo con la sustancia de interés y permiten detectar el posible daño al ADN y los resultados pueden generalizarse para dividir la biodiversidad animal y vegetal como un modelo. Además, la aberración estructural y las alteraciones cromosómicas numéricas se pueden visualizar directamente. Además, Allium cepa es distintivo en cuanto a su eficiencia en comparación con otros bioensayos, buena correlación con otros sistemas de prueba, fácil manejo, bajo costo y tiene un tamaño ideal y un número de cromosomas $(2 \mathrm{n}=16)$. La prueba de Allium cepa tiene una sensibilidad similar a la de los ensayos de carcinogenicidad en algas, linfocitos humanos y en roedores mostraron una correlación del $82 \%$ con Allium cepa ${ }^{38}$.En virtud 
de que no hay estudios de estas sustancias químicas, en células vegetales, el objetivo es comprobar el efecto genotóxico de la AA y GA en células de Allium cepa.

\section{Materiales y métodos}

\section{Químicos}

La acrilamida (AA; número de registro CAS: 7906-1, >/- 99,5 \% puro) se adquirió de Fluka (Buchs, Switzerland) y la glicidamida (GA número de registro CAS: 5694-00-8, > 98,5\% puro, conteniendo aproximadamente $1 \%$ AA) se obtuvo de Toronto Research Chemicals (North York, Ontario, Canadá)

\section{Bulbos y raíces adventicias}

Fueron utilizados bulbos de cebolla variedad perla de $20 \mathrm{~g}$ de peso. Las raíces adventicias fueron obtenidas colocando las bases de los bulbos en tubos de vidrios con agua filtrada y equipado con un equipo de burbujeo de aire (10 $-20 \mathrm{ml} / \mathrm{min}$ ). Todo esto en estufa y en oscuridad a $25^{\circ} \mathrm{C}+/-0,5{ }^{\circ} \mathrm{C}$. Los bulbos control fueron incubados en el agua filtrada. Las concentraciones de los compuestos químicos ensayadas en este trabajo (0, 50, 100 y $250 \mathrm{uM})$, surgen de los estudios preliminares de toxicidad realizados. Se administró como solución de AA y GA en las concentraciones antes mencionadas de estos elementos, preparados con el mismo tipo de agua. Todas se administraron cuando las raíces tenían $2-3 \mathrm{~cm}$ de largo. La solución se renovó cada $24 \mathrm{~h}$, siguiendo la metodología aplicada por Lerda ${ }^{39}$.

\section{Medición del crecimiento de la raíz}

El crecimiento se determinó midiendo la longitud de 10-12 raíces previamente identificadas por bulbo, cada $24 \mathrm{~h}$ durante $96 \mathrm{~h}$.

\section{Actividad proliferativa}

La actividad proliferativa se cuantificó determinando la frecuencia celular mitótica en la punta de la raíz. Las raíces obtenidas a $0,12,24$ y $48 \mathrm{~h}$ después del comienzo del ensayo se fijaron en etanol-acético, $3: 1 \mathrm{v} / \mathrm{v}$ a $4^{\circ} \mathrm{C}$ durante 24 h. Después de teñir las raíces con ácido acético-clorhídrico ${ }^{40}$, el área meristemática se aplastó y la frecuencia de las células mitóticas se determinaron en 1000 células aplastadas.

\section{Aberraciones cromosómicas}

Cuando las raíces tenían $3-5 \mathrm{~cm}$ de largo, se expusieron a la concentración de AA y GA $(0,50,100,250 \mathrm{uM})$ durante $48 \mathrm{~h}$. Luego, estuvieron expuestos a la acción de la colchicina al $0.1 \%$ durante $3 \mathrm{~h}$. Se cortaron las raíces y se fijaron en etanol-ácido acético (3: $1 \mathrm{v} / \mathrm{v})$ a $4{ }^{\circ} \mathrm{C}$ durante $24 \mathrm{~h}$. Posteriormente, se tiñeron con orceína acética. Se puntuaron aproximadamente 5000 células por frecuencia y tipo de aberraciones cromosómicas. Se utilizó etilmetilsulfóxido (EMS) al 0,2\% como control positivo. La frecuencia (porcentaje) de células aberrantes se determinó sobre la base del total de células contadas y el número de células en división. Para determinar diferencias significativas entre las raíces tratadas y las de control, se utilizó el ensayo de Irwin-Fischer (Z) para la probabilidad exacta.

\section{Resultados}

Se analizó el efecto de diferentes concentraciones de AA y GA en el crecimiento longitudinal de la raíz. Se observó que la GA a la concentración de $250 \mathrm{uM}$ detiene el proceso de crecimiento después de $24 \mathrm{~h}$, sin embargo, esto se debe a la muerte de la raíz. La AA a la misma concentración no detiene el crecimiento de la raíz y a las concentraciones de 0,50 y $100 \mathrm{uM}$ de AA y GA, la tasa de crecimiento de la raíz se reduce en comparación con las raíces de control. Estos hallazgos confirman que la GA causa una inhibición del crecimiento de la raíz cuya intensidad depende de la concentración en un medio dado (Figura.1).

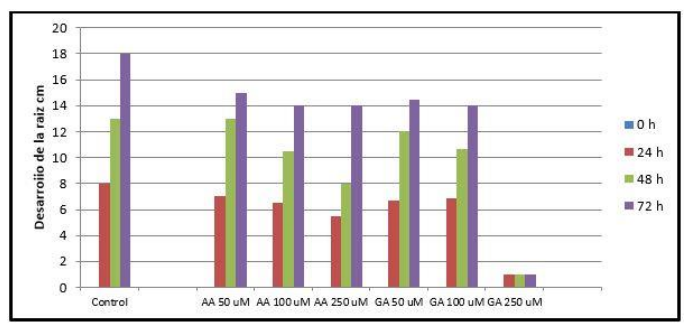

Figura 1. Efecto de la acrilamida y glicidamida sobre el desarrollo de la raíz.

En cuanto a la proliferación celular, este estudio mostró un comportamiento dispar entre AA y GA. Mientras que la frecuencia de las células mitóticas se redujo progresivamente al aumentar las concentraciones de GA ( $250 \mathrm{uM})$, los valores mínimos se alcanzan después de $24 \mathrm{~h}$. Esta inhibición fue transitoria, ya que se observó la recuperación de la actividad de proliferación después de $48 \mathrm{~h}$ de incubación con GA. A la 
concentración de 250 uM la AA se comportó como los bulbos control. Este hallazgo sugiere que la GA bloquea el ciclo de división celular en una etapa previa a la mitosis. Los bulbos de cebolla expuestos a 0,50 y $100 \mathrm{uM}$ de AA y GA tenían una frecuencia de células mitóticas similar a la de los bulbos de control (Figura. 2).

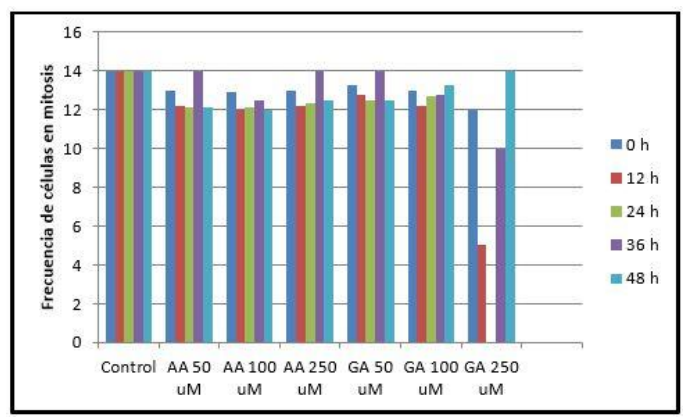

Figura 2. Efecto de la acrilamida y la glicidamida sobre la actividad proliferativa en la región meristemática de la raíz.

La GA induce efectos clastogénicos en las raíces meristemáticas de Allium cepa, en cambio la AA no produce estos efectos. Los datos sobre la frecuencia de las células aberrantes se presentan en la (Tabla 1). Se observó un aumento en la frecuencia de las células aberrantes con la concentración más alta de GA (250 uM). El ensayo de Irwin-Fischer $(Z)$ para la probabilidad exacta se realizó con el total de células aberrantes, ya que a la concentración de $250 \mathrm{uM}$ solo se produjeron puentes y células binucleadas, por lo que diferían del control positivo. El resultado fue $\mathrm{Z}=6.99$ (> 1.90) significativo en $\mathrm{P}$ $<0.05$.

Tabla 1. Frecuencias de aberraciones citológicas inducidas por AA y GA en el sistema de Allium cepa.

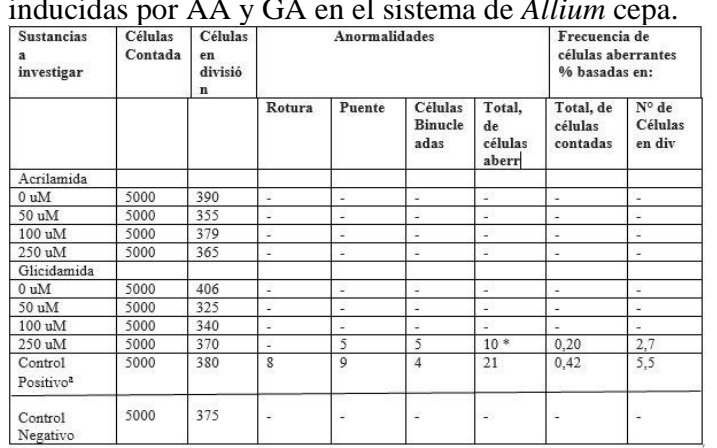

a: Etilmetilsulfóxido al 0,2\%

*: Z: 6,99 gnificativo $\mathrm{p}<0,05$

\section{Discusión}

Los hallazgos de este trabajo muestran que la AA a las concentraciones utilizadas no mostró un comportamiento similar a la GA, conocido genotóxico. La GA inhibe el crecimiento longitudinal de la raíz en función de su concentración y que también bloquea el ciclo de división celular en una etapa previa a la mitosis. Además, se determinó que los bulbos de cebolla poseen mecanismos de eliminación o neutralización de GA. Las aberraciones citológicas observadas en el ensayo de Allium a una concentración de $250 \mathrm{uM}$, mostraron que la GA era capaz de inducir la genotoxicidad a nivel de cromosoma. La mayoría de las anormalidades comunes fueron puentes. Estos probablemente se producen por la interrupción y unión de los cromosomas o las cromátidas ${ }^{41}$ o como resultado de la rigidez del cromosoma o que podría atribuirse a la translocación o la inversión desigual de los segmentos cromosómicos ${ }^{41}$. Estos hallazgos son similares a lo obtenido por Pingarilho y $\mathrm{col}^{32}$ en donde ellos utilizaron la técnica de intercambio de cromátides hermanas en células humanas. Demostraron que la AA solo indujo ligeramente intercambio de cromátides hermanas a una concentración muy alta y, por el contrario, GA es claramente genotóxico para los linfocitos humanos cultivados. Sugimura y col $^{33}$ demostraron, en el sistema bacteriano de Ames (Salmonella typhimurium), el efecto mutagénico de la GA en la superficie carbonizada de pescado y carne de res. Los resultados obtenidos en la presente investigación refuerzan la importancia de Allium cepa, ya que, en este estudio, los resultados son similares a los obtenidos con otros bioensayos. También es importante señalar, como ha sido mencionado por Lerda ${ }^{42-43}$, que incluso si el metabolismo de la planta es diferente, Allium cepa muestra excelentes resultados de análisis citotóxicos, y que la observación de las alteraciones cromosómicas en las células vegetales es fácil de visualizar. Esta especie de planta se ha utilizado como un indicador para advertir a las personas sobre el consumo de ciertos alimentos ${ }^{44-45}$.

A partir de estos resultados sobre la genotoxicidad de la AA, hallados por otros autores en células humanas, bacterias y este, en células vegetales, permitiría continuar con estudios epidemiológicos, que hasta la fecha no se ha publicado ninguno que afirme que las cantidades de acrilamida consumidas en los alimentos, por la población en general, aumenten significativamente el riesgo de sufrir algún tipo de cáncer por esta causa. Por otra parte, hasta el momento no hay estudios de AA y GA en células vegetales a los efectos de establecer una comparación que permita reforzar lo hallado en este trabajo. 
Las limitaciones que puede presentar este ensayo esta dado en las condiciones experimentales, como la concentración de oxígeno y la composición del agua en la que crecen las raíces.

\section{Conclusiones}

En este estudio, aunque las concentraciones de AA y GA son mucho más altas que lo niveles de exposición alimentaria en humanos, el GA fue claramente genotóxico para células vegetales, a una determinada concentración, con una marcada citotoxicidad. Esto demostrado por las variables analizadas como el crecimiento radicular, la actividad proliferativa y las aberraciones cromosómicas. Por lo tanto, nos permitió distinguir las diferencias entre AA y GA. Este enfoque es crucial, no solo para una profunda comprensión de la utilidad de la técnica de Allium en la evaluación de riesgos, sino también para la identificación de genotipos que modulan potencialmente el daño genotóxico, en un escenario de exposición a la AA.

\section{Bibliografía}

1. Jägerstad, M and Skog, K (2005) Genotoxicity of heat-processedfoods. Mutat. Research 574:156-17

2. International Agency for Research onCancer (1986) Someacrylamideused in plastic and elastomers. IARC monographson the evaluation of carcinogensrisk of chemicaltohumans, vol. 39. Lyon, France: IARC 403 pp.

3. LoPachin, RM (2004) The changingview of acrylamideneurotoxicity. Neurotoxicology 25(4):617-630.

4. International Agency for Research onCancer (1994) Acrylamide. IARC monographson the evaluation of carcinogensrisktohumans, some industrial chemical 60:387-433.

5. Reportfrom the ScientificCommittee of NorwegianFood Control Authority (SNT) (2002) Riskassessment of acrylamideintakefromfoodswithspecial emphasisoncancerrisk

6. European Chemical Bureau, (2002) Toxicology and Chemical Substances. Classification and Labelling. http://ecb.jrc.it/classification-labelling
7. OMS (Organización Mundial de la Salud) (2002) Australiansurvey of acrylamide in carbohydratebasedfoods. FoodAdditContam 21(8):721-736.

8. Frankel, E.N. (1998). Photooxidation of unsaturatedfats. EnLipidoxidation, cap. 3, págs. 43-54. The oilyPress, Dundee, Escocia.

9. Morales Navas,F, MesiasGarcia,M y Delgado Andrade,C (2019) Acrilamida en alimentos. ACTA/CL 67:5 - 12.

10. Mesias,M; Nouali,A; Delgado Andrade,C; Morales, F (2020) How Far Is the Spanish Snack Sector from meeting the AcrylamideRegulation 2017/2158? Foods 9, 247:2-12 (doi:10.3390/foods9020247)

11. Parkin, K.L. (2010). Enzimas. En Química de los alimentos, cap. 6, 3ra. Edición, págs. 327-433. Editado por S. Damodaran, K.L. Parkin y O.R. Fennema, Editorial Acribia, Zaragoza, España.

12. Cheftel, J.C.; Cheftel, H. (1976). Los principales sistemas bioquímicos alimentarios - Comportamiento durante los tratamientos. En Introducción a la bioquímica y tecnología de los alimentos, vol. I, cap. II, págs. 41-235. Editorial Acribia, Zaragoza.

13. Perla, V.; Holm, D.G.; Jayanty, S.S. (2012). Effects of cookingmethodsonpolyphenols, pigments and antioxidantactivity in potatotubers. LWT - FoodScience and Technology 45: 161-171.

14. Parkin, K.L. (2010). Enzimas. En Química de los alimentos, cap. 6, 3ra. Edición, págs. 327-433. Editado por S. Damodaran, K.L. Parkin O.R. Fennema, Editorial Acribia, Zaragoza, España.

15. Ho, C.-T.; Rafi, M.M.; Ghai, G. (2010). Sustancias bioactivas: Nutracéuticas y tóxicas. En Química de los alimentos, cap. 12, 3ra. Edición, págs. 747-775. Editado por S. Damodaran, K.L. Parkin y O.R. Fennema, Editorial Acribia, Zaragoza.

16. Lehninger, A.L. (1978). Biosíntesis de los aminoácidos y de algunos derivados; metabolismo del nitrógeno 
inorgánico. En Bioquímica, 2da edición, cap. 25, Págs. 705-738. Ediciones Omega, Barcelona.

17. Brecht, J.K.; Ritenour, M.A.; Haard, N.F.; Chism, G.W. (2010). Fisiología post-cosecha de los productos vegetales. En Química de los alimentos, cap. 17, 3ra. Edición, págs. 973-1049. Editado por S. Damodaran, K.L. Parkin y O.R. Fennema, Editorial Acribia, Zaragoza.

18. Rice,JM (2005) The carcinogenicity of acrylamide. Mutat. Res. 580:3-20.

19. E. Bergmark, Hemoglobinadducts of acrylamide and acrylonitrileinlaboratoryworkers, Chem. Res. Toxicol. 10 (1997) 78-84

20. H.L. Peres, In vivo dosimetry of someimportant industrial chemicalsbymeasurement of theirreactionproductswithhemoglobin, Doctoral thesis at the Department of Molecular Genome Research, StockholmUniversity, Stockholm, 2000 .

21. B. Paulsson, A. Rannug, A.P. Henderson, et al (2005) In vitro studies of the influence of glutationes transferases and epoxidehydrolaseon the detoxification of acrylamide and glycidamide in blood, Mutat. Res. 580: 53-59.

22. M.L. Gargas, C.R. Kirman, L.M. Sweeney, et al (2009) Acrylamide: Consideration of speciesdifferences and nonlinearprocesses in estimatingrisk and safety for human ingestion, FoodChem. Toxicol. 47:760-768.

23. Tritscher AM (2004) Human healthriskassessment of processingrelatedcompounds in food. ToxicolLett 149: 177-186.

24. Friedman M (2003) Chemistry, biochemistry and safety of acrylamide.Areview. JAgricFoodChem 51: 4504-4526.

25. Dybing E, Farmer PB, Andersen M, Fennell TR, et al (2005) Human exposure and internaldoseassessments of acrylamide in food. FoodChemToxicol 43: 365-410
26. FoodDrinkEuropeUpdatesIndustryWide AcrylamideToolbox. 2019.

27. K.M. Wilson, L.A. Mucci, B.A. Rosner, W.C. Willett, (2010) A prospectivestudyondietaryacrylamidein take and the riskforbreast, endometrial, and ovariancancers, CancerEpidemiol. BiomarkersPrev. 19: 2503-2515.

28. IARC (1994) Monographson the Evaluation of CarcinogenicRisksto Human: Some Industrial Chemicals, no. 60, International Agency for Research onCancer, Lyon, pp. 389433.

29. K.L. Dearfield, G.R. Douglas, U.H. Ehling, M.M.Moore, (1995) Acrylamide - a review of itsgenotoxicity and assessment of heritablegeneticrisk, Mutat. Res. 330 71-99.

30. H. Glatt, H. Schneider, Y. Liu, (2005) V79-hCYP2E1-hSULT1A1, a cell line for the sensitivedetection of genotoxiceffectsinducedbycarbohydrat epyrolysisproducts and otherfoodborne chemicals, Mutat. Res. 580 4152 .

31. C. Martins, N.G. Oliveira, M. Pingarilho, C.G. Gamboa et al (2007) Cytogeneticdamageinducedbyacrylami de and glycidamide inmammaliancells: correlationwithspecificglycidamideDNA adducts, Toxicol. Sci. 95: 383390.

32. Pingarilho,M; Oliveira,N; Martins,C; Costa Gomes,B. et.al (2013) Induction of sisterchromatid Exchange byacrylamide and glycidamide in human lymphocytes: Role of polymorphisms in detoxification and DNA-repair genes in the genotoxicity of glycidamide. Mutat.Res 752:1- 79.

33. Sugimura, T; Kawachi, T; Nagao, M: Yahagi,T; (1977) Mutagenicprinciples in tryptophan and phenylalanineProc.Japan.Acad 53: 5861.

34. L. Abramsson-Zetterberg, (2003) Thedose-response relationship at verylow doses of acrylamideis linear in the flowcytometer-based mouse micronucleusassay, Mutat. Res. $535: 215-222$ 
35. Leme, D.M., Marin-Morales, M.A., (2009). Allium cepa test in environmentalmonitoring: A reviewonitsapplication.

MutationResearch/Reviews

in

Mutation Research 682, 71-81.

36. Paz, M., Muzio, H., Mendelson, A., Magdaleno, A. (2006). Evaluation of genotoxicity and toxicity of Buenos Aires city hospital wastewatersamples. Journal of the BrazilianSociety of Ecotoxicology 1, 1-6.

37. Gomurgen AN

Cytologicaleffect of the potassium metaᄀ bisulphite and potassoiumnitratefoodpreservativeonro ottips of Allium cepa L. Cytologia 70: $119-128$

38. Turkoglu S (2007) Genotoxicity of fivefoodpreservativestestedonroottips Allium cepa L. Mutat Res 626: 4-14.

39. Lerda D (1992) The effect of lead on Allium cepa L. Mutat Res 281:89-92.

40. Jio T, Joe H, Levan A, Stalfelt MG (1950) The use of oxyquinoline in chromosomeanalysis. Withappendix: The effect of oxyquinolineonprotoplasmiviscosity. An Estac Exp Aula Dei 2: 21-64.

41. Rank, J., Nielsen, M., (1994). Evaluation of the Allium anaphasetelophase test in relationtogenotoxicity screening of industrial wastewater.Mutation Research/EnvironmentalMutagenesisa nd RelatedSubjects 312, 17-24.
42. Lerda D (1992) The effect of lead on Allium cepa L. Mutat Res 281: 89-92.

43. Lerda D, BiagiBistoni M, Pelliccioni $\mathrm{P}$, Litterio N (2010) Allium cepa as a biomonitor of ochratoxin A toxicity and genotoxicity. PlantBiol (Stuttg) 12: 685- 688 .

44. Lerda D, Biaggi B, Peralta N, Ychari S et.al (2005 Fmonisins in foodfromCordoba (Argentina), presence and genotoxicity. FoodChemToxicol 43: 691-698.

45. Lerda D, Miotti E, Litterio N (2017) Detection Genotoxicity of Ochratoxin A (OTA) in Raisins. EuropeanScientificJournal 13:1-8.

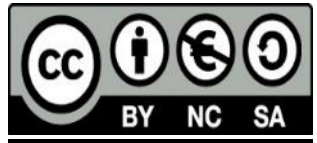

